\title{
Extensive in silico analysis of Mimivirus coded Rab GTPase homolog suggests a possible role in virion membrane biogenesis
}

\author{
Amrutraj Zade, Malavi Sengupta and Kiran Kondabagil * \\ Department of Biosciences and Bioengineering, Indian Institute of Technology-Bombay, Mumbai, India
}

\section{OPEN ACCESS}

Edited by:

Bruno Pozzetto,

University Jean Monnet of

Saint-Etienne, France

Reviewed by:

Takashi Irie,

Hiroshima University, Japan

Yael Mutsafi,

Weizmann Institute of Science, Israel

*Correspondence:

Kiran Kondabagil,

Department of Biosciences and Bioengineering, Indian Institute of

Technology-Bombay, Adi

Shankaracharya Marg, Powai,

Mumbai-400076, India

kirankondabagil@iitb.ac.in;

kirankondabagil@gmail.com

Specialty section:

This article was submitted to

Virology, a section of the journal

Frontiers in Microbiology

Received: 17 April 2015

Accepted: 24 August 2015

Published: 15 September 2015

Citation:

Zade $A$, Sengupta $M$ and Kondabagil K (2015) Extensive in silico analysis of Mimivirus coded Rab

GTPase homolog suggests a possible role in virion membrane biogenesis.

Front. Microbiol. 6:929

doi: 10.3389/fmicb.2015.00929
Rab GTPases are the key regulators of intracellular membrane trafficking in eukaryotes. Many viruses and intracellular bacterial pathogens have evolved to hijack the host Rab GTPase functions, mainly through activators and effector proteins, for their benefit. Acanthamoeba polyphaga mimivirus (APMV) is one of the largest viruses and belongs to the monophyletic clade of nucleo-cytoplasmic large DNA viruses (NCLDV). The inner membrane lining is integral to the APMV virion structure. APMV assembly involves extensive host membrane modifications, like vesicle budding and fusion, leading to the formation of a membrane sheet that is incorporated into the virion. Intriguingly, APMV and all group I members of the Mimiviridae family code for a putative Rab GTPase protein. APMV is the first reported virus to code for a Rab GTPase (encoded by R214 gene). Our thorough in silico analysis of the subfamily specific (SF) region of Mimiviridae Rab GTPase sequences suggests that they are related to Rab5, a member of the group II Rab GTPases, of lower eukaryotes. Because of their high divergence from the existing three isoforms, A, B, and C of the Rab5-family, we suggest that Mimiviridae Rabs constitute a new isoform, Rab5D. Phylogenetic analysis indicated probable horizontal acquisition from a lower eukaryotic ancestor followed by selection and divergence. Furthermore, interaction network analysis suggests that vps34 (a Class III PI3K homolog, coded by APMV L615), Atg-8 and dynamin (host proteins) are recruited by APMV Rab GTPase during capsid assembly. Based on these observations, we hypothesize that APMV Rab plays a role in the acquisition of inner membrane during virion assembly.

Keywords: NCLDV, APMV, Rab GTPase, subfamily specific (SF) region, membrane acquisition, virus assembly

\section{Introduction}

With a particle size of about $750 \mathrm{~nm}$, Acanthamoeba polyphaga mimivirus (APMV) is one of the largest viruses known so far (La Scola et al., 2003; Claverie et al., 2006). APMV belongs to the monophyletic clade of large eukaryotic DNA viruses known as the nucleo-cytoplasmic large DNA viruses (NCLDV) (Iyer et al., 2001). In the mature APMV particle, its $1.2 \mathrm{Mbp}$ linear genome is encapsidated within the icosahedral capsid that is underlined by a lipid bilayer (Xiao et al., 2005). Acquisition of inner viral membrane is a critical step during the capsid assembly and is poorly understood. 
APMV is a cytoplasmically replicating virus that carries out viral genome replication transcription, protein synthesis and the subsequent stages of particle formation and virus budding in the giant cytosplasmic structures known as viral factories. Viral factories are formed at $4 \mathrm{~h}$ post infection (PI) and the budding of new viral particles has been observed at around $8 \mathrm{~h}$ PI (Suzan-Monti et al., 2007; Zauberman et al., 2008). The membrane biogenesis of APMV is initiated from the host cytoplasmic membrane cisternae at around $7.5 \mathrm{~h}$ PI (Zauberman et al., 2008; Mutsafi et al., 2013). Regular budding of $\sim 70 \mathrm{~nm}$ vesicles from the cellular cisternae have been observed around the viral factories at earlier PI times (Mutsafi et al., 2013). Continuous fusion of smaller vesicles leads to the formation of multivesicular bodies followed by its fissure to form large membrane sheets that are incorporated into the viral progenies (Mutsafi et al., 2013). The major capsid protein L425, a homolog of vaccinia virus D13, acts as a scaffolding protein and initiates capsid assembly above the membrane sheet. A consistent membrane overhang that prevents the premature closure of the capsid is trimmed off upon completion of the capsid assembly, leaving a $\sim 20 \mathrm{~nm}$ nonvertex transient opening for genome packaging (Mutsafi et al., 2013).

Rab GTPases, a subfamily of small GTP binding proteins within the Ras superfamily, are the key regulators of membrane trafficking (Bourne et al., 1990). Rab GTPase functions by alternating between two states; a GTP-bound active state in which it interacts with effector proteins and a GDP-bound inactive state in which it interacts with proteins like Rab escort protein (REP) and GDP dissociation inhibitor (GDI) (Lee et al., 2009). Functionally divergent Rab GTPases are involved in budding and scission of membrane vesicles from donor organelles, their transport along the actin and microtubules, association with target membrane through tethering complex, and finally their fusion with recipient organelle (Zerial and McBride, 2001). Many intracellular pathogens reside in the vacuoles and extensively modify its host-derived membrane by either recruiting or excluding surface Rab proteins with the help of pathogen proteins (Brumell and Scidmore, 2007; Kumar and Valdivia, 2009). Aside from Legionella pneumophila, which encode proteins that directly interact with Rab, other Rab-mediated mechanisms employed by bacterial pathogens remain poorly understood (Brumell and Scidmore, 2007).

Rab GTPases are the hallmarks of the eukaryotic endomembrane system that are found in prokaryotes. It is quite intriguing for a virus, which does not have a cellular structure, to code for this particular protein. In this report, we present a thorough sequence, structural, and phylogenetic analysis of Rab GTPases of Mimiviridae Group I viruses. Our results indicate that Mimiviridae Rabs belong to Rab5 family and were probably acquired horizontally from an early unicellular eukaryote. Further, sequence and structural comparisons suggest that Mimiviridae Rabs possess all the signature motifs of family Rab5, and have probably diverged to form a new isoform, 5D. Considering the above observations and the available APMV transcriptome data and membrane assembly insights (Legendre et al., 2011; Mutsafi et al., 2013), we speculate that the APMV Rab GTPase might play a role in the acquisition of inner membrane during capsid assembly.

\section{Materials and Methods}

\section{Retrieval of Rab Sequences}

The putative Rab GTPase amino acid sequences from Mimiviridae family viruses were retrieved from UniprotKB. The APMV R214 sequence was used as a query to search for homologous sequences in the $\mathrm{nr}$ (nonredundant) GenBank protein database. Sequence similarity searches were performed using the BLASTP application (http://blast.ncbi.nlm.nih.gov/ Blast.cgi) with standard settings except the maximum target sequences menu under General Algorithm parameters was changed to 250 from 100 . Redundant, unnamed and hypothetical sequences were removed before the alignment. A dataset for comparative analysis was prepared using all the annotated Rab GTPase isoforms from Homo sapiens, Plasmodium falciparum, Caenorhabditis elegance, Drosophila melanogaster, Trichomonas vaginalis, and Saccharomyces cerevisiae.

\section{Sequence Analysis, Conserved Motif Identification, and Phylogenetic Reconstruction}

Mimiviridae Rab GTPase sequences were subjected to multiple sequence alignment using Clustal Omega (Sievers et al., 2011) with default settings. The alignment was subjected to the ESPript 3.0 (Gouet, 2003) and secondary structure was predicted using PSIPRED (McGuffin et al., 2000) and Jpred3 servers (Cole et al., 2008).

Phylogenetic reconstruction was done in MEGA6 (Molecular Evolutionary Genetics Analysis) program (Tamura et al., 2013). Sequences were aligned using ClustalW with BLOSUM62 as weight matrix. Neighbor-Joining (NJ) method based trees were generated with complete deletion and p-distance options employed in MEGA6. Trees were analyzed using Bootstrap method for 1000 iterations (Nei and Kumar, 2000). MEGA6 was also used for visual details and representation.

\section{Structure Prediction and Alignment}

APMV R214 sequence was threaded to I-TASSER online server (Zhang, 2008) against the I-TASSER template database for homology based structural modeling. Structure with the best Cscore of 0.59 , was selected for further analysis. Homo sapiens Rab5b (HsRab5b) structure was retrieved from PDB database (2HEI). Predicted APMV Rab and HsRab5b structures were aligned using PyMol (DeLano, 2002).

\section{Interaction Network}

APMV Rab interaction network was prepared using the Cytoscape platform (Saito et al., 2012). P. falciparum was used as the source organism to generate APMV Rab interactome. $P$. falciparum Rab5B (PfRab5B) physical interaction network was retrieved from StringDB and homologous protein sequences were obtained from UniprotKB database. Interacting species were used to search for their homologs using multiple reciprocal BLASTP at NCBI (http://blast.ncbi.nlm.nih.gov/Blast.cgi) against either APMV or Acanthamoeba castellanii str. Neff sequence datasets. Orthologs with $E<0.05$ were selected and used to build the physical interaction network. 


\section{Results}

\section{All Members of the Group I Mimiviridae Family Encode for Rab GTPase}

A thorough BLASTP search of the NCBI GenBank database, with APMV R214 gene, that was annotated as a probable small GTPase protein (Raoult et al., 2004) as the query sequence against all members of the NCLDV superfamily, revealed that only Moumouvirus, Mamavirus, Lentille virus, Hirudovirus, Courdo virus, Samba virus, and Megavirus code for R214 homolog (Table 1). Interestingly, all seven viruses belong to the group I of Mimiviridae family of NCLDVs. A phylogenetic tree, constructed using the DNA polymerase $\mathrm{B}$, with representative sequences from all NCLDVs, suggests that Rab GTPase was acquired by an ancestor of group I Mimiviridae lineages (Figure 1).

\section{R214 Gene Product and its Mimiviridae Group I Homologs are Rab GTPases}

The signature Rab GTPase motifs, the PM1 motif or the ploop with a consensus GxxxxGKS/T and, PM2 and PM3 motifs, with conserved sequences $\mathrm{T}$ and DxxGQ, respectively, are strictly conserved in all three lineages of Mimiviridae (Figure 2A). The G1, G2, and G4 motifs, with consensus F, ANKxD, N, respectively are conserved, while the G3 motif is represented by either SSF or NCI (Figure 2A). Mimiviridae Rab GTPase possess all the conserved five Rab-family specific motifs, RabF1RabF5, clustered around the switch regions I and II (Figure 2A). The first motif RabF1, represented by a consensus IGAAF in the Mimiviridae family, is critical for facilitating the crosstalk between the switch I and switch II residues (Dumas et al., 1999). The other four motifs, RabF2-RabF5, although show some variations, are readily identifiable and are present in the same structural context, between $\beta 3$ and $\beta 4$ sheets, and are closer to or part of the switch II region as in the well-known Rabs (Figure 2A) (Pereira-Leal and Seabra, 2000). Furthermore, the hypervariable region of Mimiviridae Rab is situated at the carboxy-terminal to the GTPase-fold that is followed by the CAAX boxes ( $\mathrm{C}$, cys; $\mathrm{A}$, aliphatic; $\mathrm{X}$, any amino acid). The CAAX boxes are the signature prenylation motif of Rabs that

TABLE 1 | Rab GTPases in Mimiviridae family.

\begin{tabular}{|c|c|c|c|}
\hline Lineage & Virus & Gene Name & UniProt ID \\
\hline \multirow[t]{5}{*}{ A } & Acanthamoeba polyphaga mimivirus & MIMI_R214 & Q5UQ27 \\
\hline & Samba virus & $\mathrm{N} / \mathrm{A}$ & W6GH08 \\
\hline & Hirudovirus strain Sangsue & HIRU_S752 & V5L429 \\
\hline & Acanthamoeba polyphaga lentillevirus & R167 & J3IZ12 \\
\hline & Acanthamoeba polyphaga mamavirus & MAMA_R280 & G8ECS6 \\
\hline \multirow[t]{3}{*}{ B } & Moumouvirus goulette & glt_00705 & M1NN89 \\
\hline & Acanthamoeba polyphaga moumouvirus & Moumou_00240 & L7RBD8 \\
\hline & Moumouvirus monve & mv_L827 & H2EFX7 \\
\hline \multirow[t]{4}{*}{ C } & Megavirus chiliensis & Mg314 & G5CS79 \\
\hline & Megavirus iba & LBA_00299 & L7Y3B3 \\
\hline & Megavirus courdo11 & CE11_00318 & K7YVK8 \\
\hline & Megavirus courdo7 & c7_R350 & H2EAJ3 \\
\hline
\end{tabular}

consists of two cysteine residues within the five residue stretch at the extreme $\mathrm{C}$-terminus in one of the following combinations, XXXCC, XXCCX, XCCXX, CCXXX, or XXCXC (PereiraLeal and Seabra, 2000). In Mimiviridae Rabs, the prenylation motif shows dimorphism as XXXCC and XXCXC (Figure 2A). Rab GTPases undergo prenylation through the attachment of geranylgeranyl moieties to these two cysteine residues that regulate the membrane insertion (Gomes et al., 2003).

Rab subfamilies are further distinguished based on the presence of subfamily specific conserved sequences (RabSF14) that show higher homology within the subfamily (Moore et al., 1995). The RabSF1-4 regions in the Mimiviridae family are also identified corresponding to sequences upstream of PM1, $\alpha 1 /$ loop3, $\alpha 3 /$ loop7 and $\alpha 5$, respectively (Figure 2A). To delineate the isoform-type of Mimiviridae Rabs that could give clues about their function, an alignment of only SF regions of Rab isoforms A-C with Mimiviridae Rabs was generated (Figure 2B). While the key residues in the SF1 and SF3 regions of Mimiviridae Rabs and Rab isoforms A-C are conserved, the residues in the SF2 and SF4 regions showed higher divergence from all other Rab5 isoforms (Figure 2B).

\section{Predicted Structure of Mimiviral Rab}

The GTPase-fold consisting of five $\alpha$-helices flanking a sixstranded $\beta$-sheet, five parallel and one antiparallel, is a common feature of the Ras superfamily (Dumas et al., 1999). These structural features were also identified in the predicted APMV Rab GTPase structure (Figure 2C). Motifs responsible for binding GTP and $\mathrm{Mg}^{2+}$ are located in the loop regions between

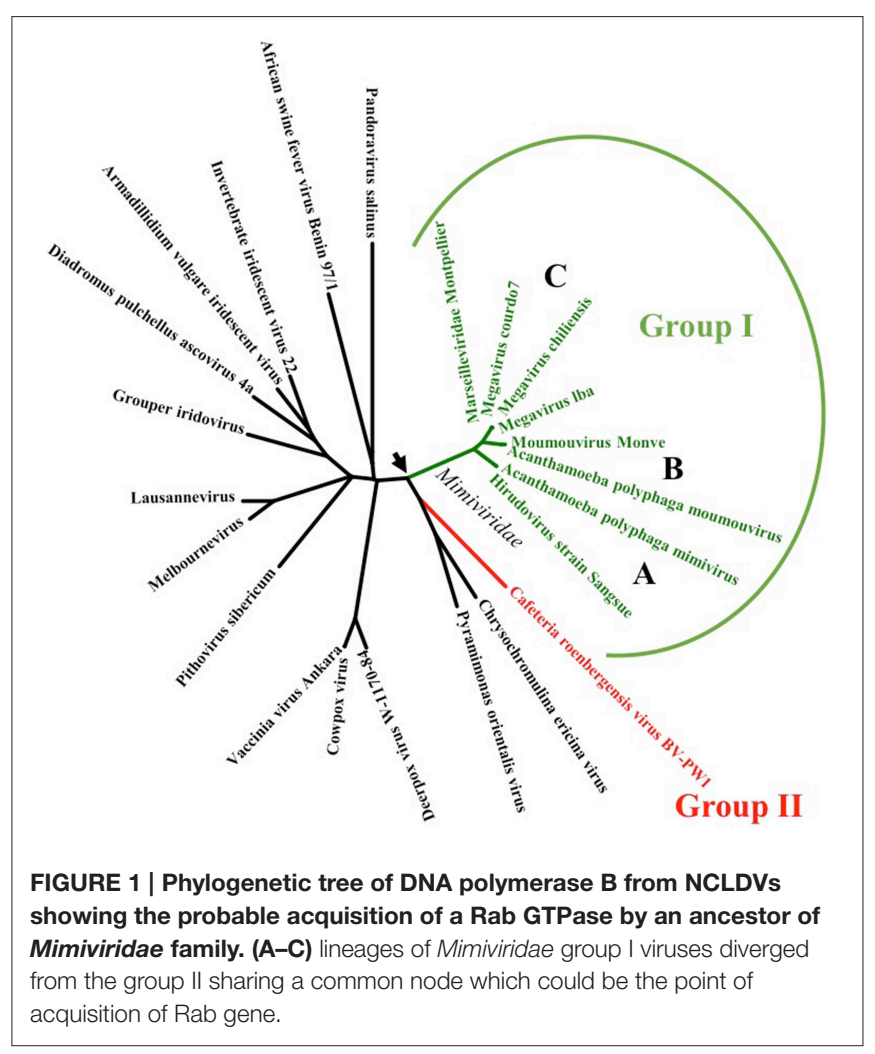




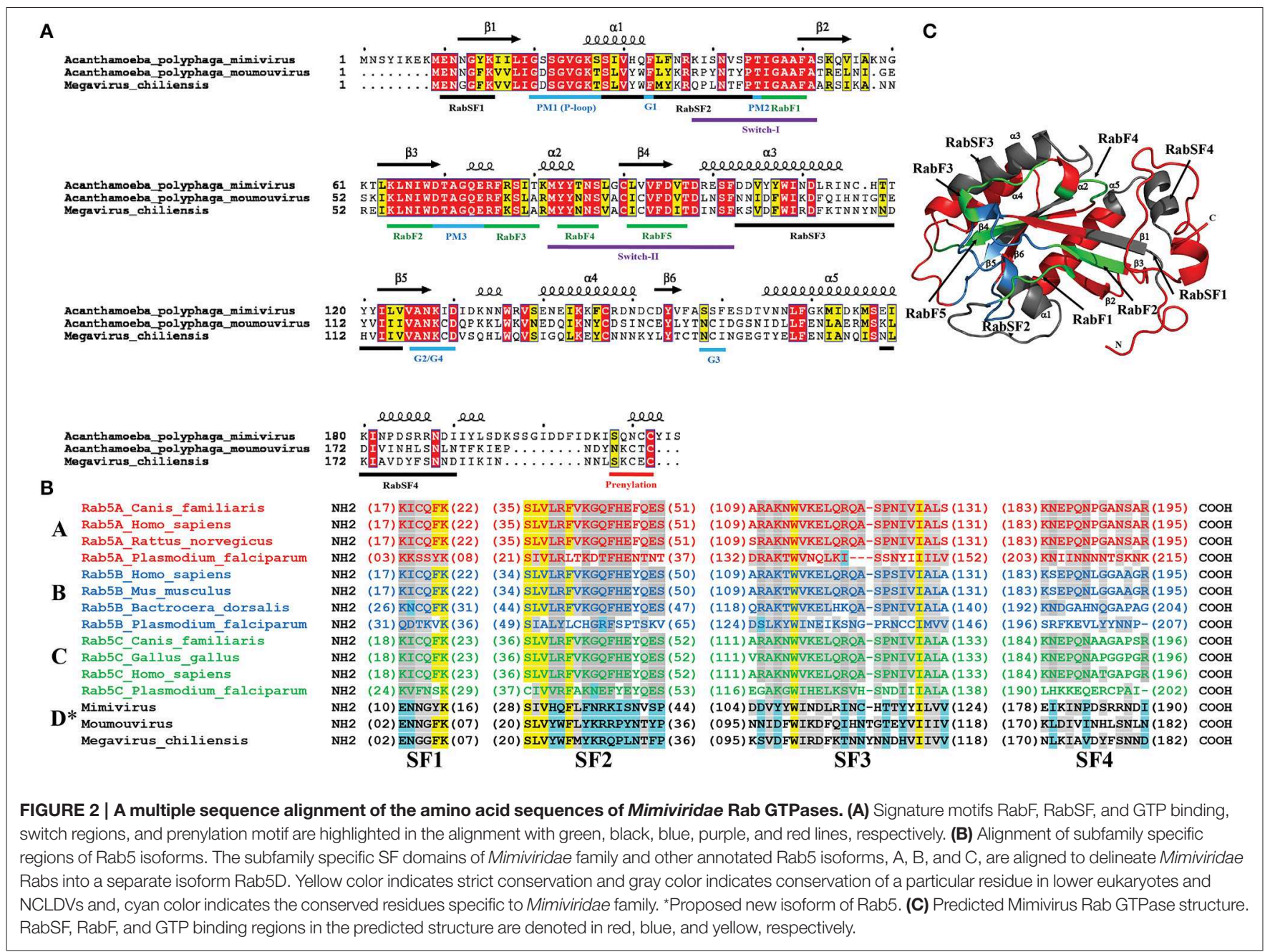

$\alpha$-helix and $\beta$-strands as seen in all the Ras superfamily proteins. The nucleotide-dependent Rab functions are primarily determined by the switch I and II regions. Solved structures indicate that the GDP bound state tends to have switch regions disordered, which upon binding to GTP, transmutes into a wellordered structure (Stroupe and Brunger, 2000). The $\gamma$-phosphate of GTP forms contact with switch I and II regions (Lee et al., 2009). The switch I and II regions in the predicted APMV $\mathrm{Rab}$ structure are located in the loop2 and loop4- $\alpha 2$-loop5, respectively (Figure 2A).

\section{Mimiviral Rab GTPase has Diverged Early from Lower Eukaryotic Rab5 and Belongs to Group II Family of Rab GTPases}

Homology search using APMV Rab GTPase retrieved Rab homologs in eukaryotes ranging from unicellular Paramecium to multicellular organisms like Homo sapiens and, different Rab families like Rab5, Rab11, and Rab4. A neighborjoining phylogenetic tree of Mimiviridae Rab with homologs showed their close association with the lower eukaryotic Rabs, specifically, protozoan Rab GTPases, including a Acanthamoeba castellanii str. Neff Rab (Figure 3A). This analysis is consistent with an earlier report that suggested that the APMV R214 gene is closely related to a homolog found in its host amoeba (Moreira and Brochier-Armanet, 2008). Furthermore, the phylogeny showed two distinct clades; one consisting of Mimiviridae family Rab, Plasmodium Rab5B, Acanthamoeba castellanii str. Neff Rab5, Trichomonas RabD1; and the other consisting of Rab4 and Rab11 from a diverse group of organisms (Figure 3A). To further extend this analysis, all annotated Rab sequences from few representative organisms including Mimiviridae Rabs were used to generate a comprehensive phylogenetic tree and found that Rabs from Mimiviridae, Plasmodium and few lower eukaryotes form a separate clade (Figure 3B).

\section{Interaction Network}

Interestingly, APMV Rab and Plasmodium falciparum Rab5B ( $P f \mathrm{Rab5})$ are homologs and the phylogeny suggests that they share a common ancestor (Figure 3A). The physical interactome of Plasmodium Rab5B was constructed using the yeast interaction network as a template and the predicted interaction network was experimentally validated (Rached et al., 2012). For example, one of the proteins that was predicted, a Plasmodium falciparum coded casein kinase1 ( $P f C K 1)$, showed interaction with only $P f$ Rab5B, but not with other PfRab5 isoforms (Rached et al., 2012). We constructed the global physical interactome of APMV 


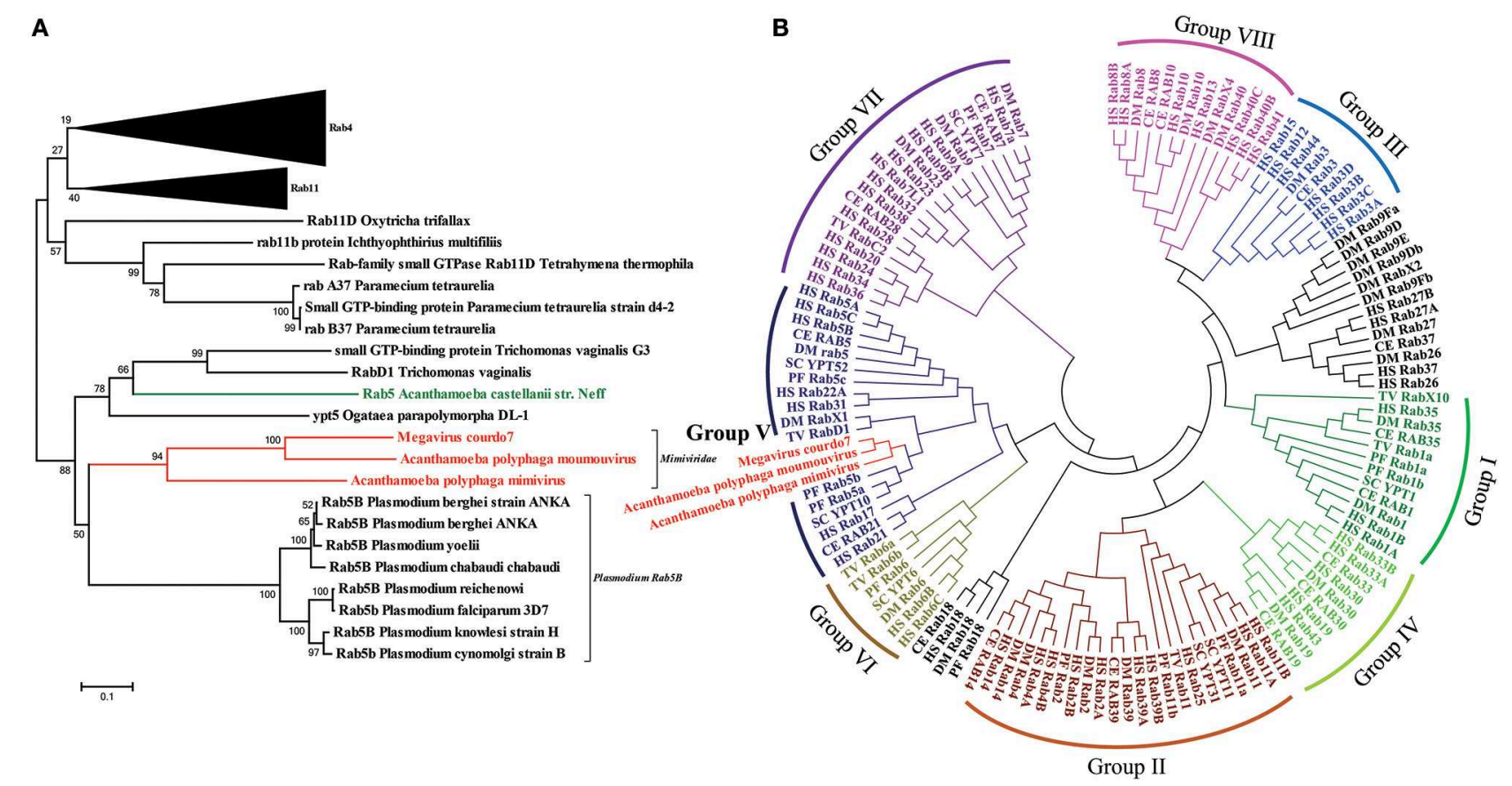

FIGURE 3 | Phylogenetic analysis. (A) Neighbor-Joining phylogenetic tree of the amino acid sequences obtained through BLASTP results using APMV Rab as a query. Mimivridae family sequences are highlighted in red and Acanthamoeba castellani str.Neff sequence is highlighted in green. (B) Neighbor-Joining phylogenetic tree constructed from a dataset comprising of the amino acid sequences of the full Rab sequences of the following representative organisms: Homo sapiens (HS), Plasmodium falciparum (PF), Caenorhabditis elegans (CE), Drosophila melanogaster (DM), Trichomonas vaginalis (TV), Saccharomyces cerevisiae (SC). Group I Rabs are shown in green, Group II in maroon, Group III in blue, Group IV in lime green, Group V in navy blue, Group VI in olive green, Group VII in purple, Group VIII in pink and unclassified Rabs are shown black, and viral sequences are shown in red.

Rab GTPase using Plasmodium Rab5B interaction network as a template (Figure 4). The interactome predicted interacting partners from both APMV and its host Acanthamoeba. Some of the important interactors identified are autophagy-related protein 8 (Atg-8), dynamin (both are host factors) and phosphoinositide-3-kinase (PI3K), coded by APMV (Figure 4). We speculate that these proteins, along with Rab GTPase, are the key players of membrane remodeling during APMV capsid assembly and their probable functions are discussed below.

Atg8: An Important Player for Membrane Enlargement Atg- 8 is a ubiquitin-like protein that tethers to the membrane through its interaction with phosphatidylethanolamine (PE) (Nakatogawa et al., 2007). Atg-8 is most likely to be recruited from the host as it was not found in the APMV genome. The membrane-tethering and hemi-fusion activities of Atg-8 are pivotal for membrane expansion (Nakatogawa et al., 2007). Atg-8 also plays an important role in the enlargement of autophagosomal membranes during autophagosome formation (Mizushima et al., 1998; Ichimura et al., 2000).

\section{Dynamin: Helical Scissors for Vesicle Scission}

Dynamin, another protein recruited from the host, plays an important role during budding of vesicles from the Trans-Golgi Network (TGN) and endosomes (Jones et al., 1998; Kreitzer et al., 2000). Dynamin family members bind to and oligomerize helically around inositol lipid molecules and impart a tubular membrane topology (Hinshaw and Schmid, 1995; Marks et al., 2001). Cooperative recruitment of dynamin and BAR (BinAmphiphysin-Rvs) domain proteins induces positive curvature on the membrane with the help of exoskeleton/endoskeleton during vesicle scission (McMahon and Gallop, 2005).

\section{Class III PI3K or Vps34: A Component of the Autophagosome Vesicle Formation Complex}

A Class III PI3K (phosphoinositide 3-kinase) or Vps34 is a kinase recruited to the vesicles in cells expressing active Rab5 and was found to colocalize with Rab5 bound to the endosomal tethering protein EEV1 (Christoforidis et al., 1999; Murray et al., 2002). Our genome-wide search found a PI3K homolog in APMV coded by L615 gene that could interact with APMV Rab and bring about vesicle enlargement. Rab5 has also been shown to form a complex with Vps34 and Beclin1 proteins that is essential for autophagosome formation (Ravikumar et al., 2008).

\section{Discussion}

The NCLDV is a large, apparently monophyletic clade of viruses that consists of seven families of eukaryotic double stranded DNA viruses, namely, Poxviridae, Iridoviridae, Ascoviridae, Asfaviridae, Phycodnaviridae, Marseilleviridae, and Mimiviridae (Iyer et al., 2001, 2006; Yutin and Koonin, 2009). Based on the phylogenetic reconstructions of the highly conserved genes called 


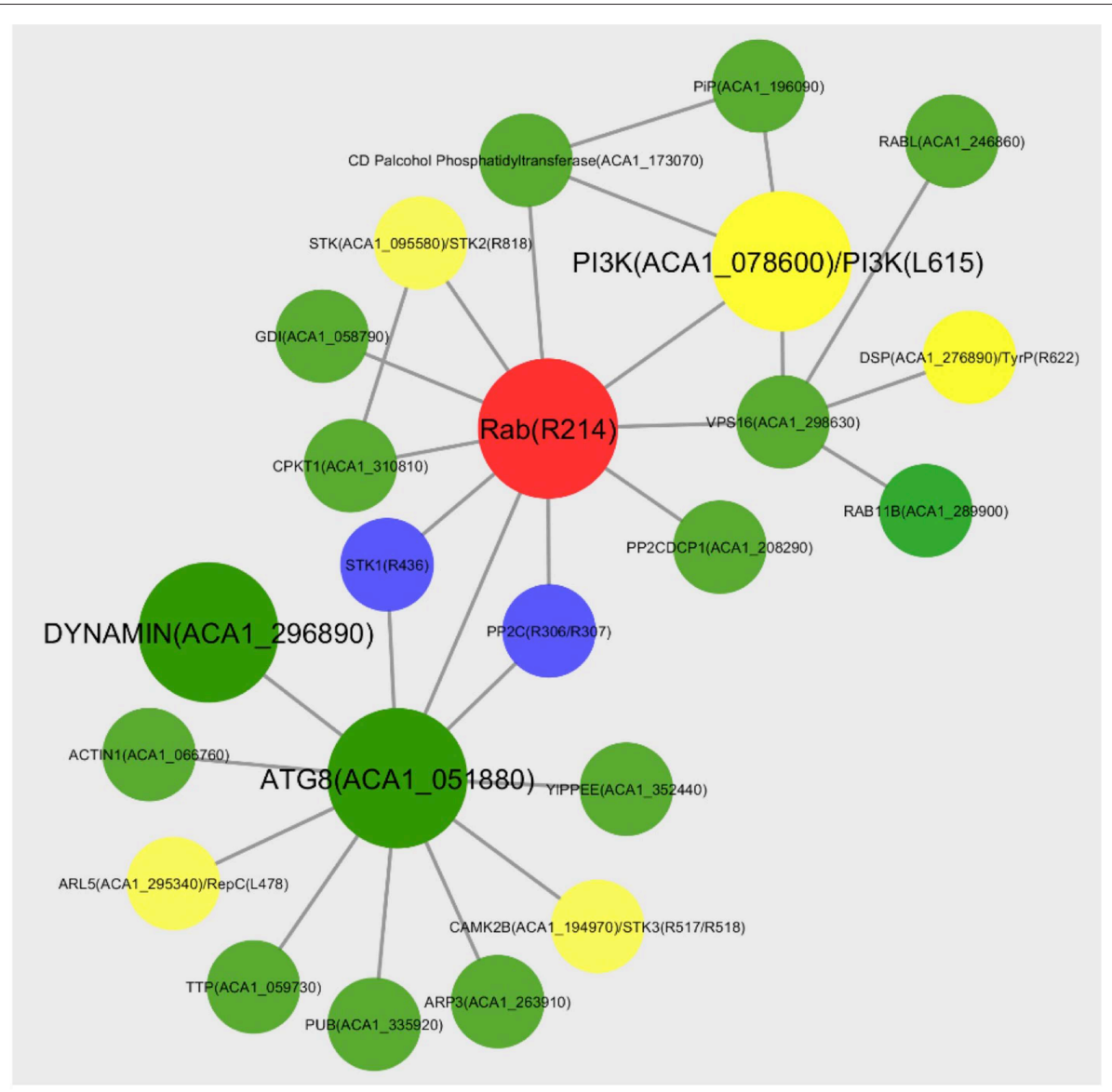

FIGURE 4 | Predicted interaction network of the APMV Rab GTPase. PfRab5b template based interaction network of Mimivirus Rab (red) physical interactome. The host proteins that are recruited are shown in green, the proteins of viral origin are shown in blue, and the proteins that could be of either host or viral origin are in yellow. The key interacting partners discussed in the text are highlighted with a bigger font.

as NCLDV clusters of orthologous groups of proteins (NCVOGs) which includes primase-helicase, DNA polymerase B, packaging ATPase and A2L-like transcription factor, Mimiviridae family has been subgrouped into two groups, I and II, and, group I Mimiviridae has been further delineated into three lineages; A, B, and C (Colson et al., 2012). Mimiviridae group I viruses possess a membrane layer underlining the icosahedral capsid (Xiao et al., 2005; Mutsafi et al., 2013). Source of this lipid bilayer and how the viruses acquire the membrane layer from endoplasmic reticulum cisternae have been demonstrated microscopically, although their molecular mechanisms remain unclear (Mutsafi et al., 2013).

All three lineages of group I Mimiviridae viruses' code for Rab GTPase (Figure 1A). Our thorough sequence analysis of Mimiviridae Rabs and the predicted structure of APMV R214 gene product suggests the presence of all the Rab signature motifs viz. $\operatorname{RabF}(1-5), \operatorname{RabSF}(1-4)$, switch regions I and II, and the classical GTP binding motifs (Figures 2A,C). The clustering of all members of Mimiviridae under the same clade, sharing a common origin with the lower eukaryotes, suggests the acquisition of Rab GTPase by a Mimiviridae ancestor from a lower eukaryotic ancestor, probably through HGT events (Figure 3A). Furthermore, a comprehensive phylogenetic analysis with all annotated Rab subfamilies indicated the close homology of Mimiviridae Rabs to the lower eukaryotic Rab5B (Figure 3B). Based on the conservation pattern in RabSF regions, Rab5 subfamily has been further divided into three isoforms, namely, 5A, 5B, and 5C (Bucci et al., 1995). Our sequence analysis showed that Mimiviridae Rabs have significant sequence divergence in the RabSF regions. This is particularly interesting since RabSF2 residues are part of the switch region I, where the nucleotide-dependent conformational changes occur (Stenmark and Olkkonen, 2001). Because the switch region residues are exposed on the surface of the molecule, it was hypothesized that they are involved in binding to effectors and regulators (Stenmark and Olkkonen, 2001). Since Rab GTPases from different species cosegregate during phylogenetic analysis, it was suggested to use phylogenetic analysis as one of the criteria, along with specific sequence in the RabSF regions, to assign Rab subfamily (Pereira-Leal and Seabra, 2001). Divergence in the RabSF region in Mimiviridae Rabs implies different functional specificity dictated by specific effector/s and regulator/s suggesting that the 
Mimiviridae Rabs could potentially form a novel subfamily of Rab5, Rab5D. Apparently, Rab group V representative, Rab5, has a complex evolutionary history (Klöpper et al., 2012). It was suggested that the Rab5 duplication has occurred independently in fungi (Ypt52), apicomplexans (Rab5B), and kinetoplastids (Rab5B) (Klöpper et al., 2012). Mimiviridae Rab BLAST search failed to retrieve other isoforms of Plasmodium Rab5 viz. A and C, but selectively retrieves only Rab5B. This observation leads to the scenario that the acquisition of Rab by Mimiviridae family might have occurred concomitantly with the duplication event in the apicomplexans and evolved to be a new isoform Rab5D.

Interestingly, superimposition of Human Rab5B bound to GDP and the predicted APMV Rab structures, although showed high structural conservation with an RMSD (Root Mean Square Deviation) value of 1.08 , the observed heterogeneity is localized in the switch regions (data not shown). The structural differences in the switch regions are the determinants of specificity toward effectors (Pfeffer, 2005; Lee et al., 2009). This observation is consistent with the RabSF sequence alignments and suggests divergence of an acquired Rab5B in the Mimiviridae family into a new isoform.

Although all known Rab5 isoforms are specialized in endosome fusion, some of them are also involved in endocytic function, endosome sorting etc. (Woodman, 2000). Studies have also shown that, besides the hypervariable region, certain $\mathrm{F}$ and SF regions are also important determinants of membrane targeting and effector protein interaction specificity (Ali and Seabra, 2005). The R214 transcript is not detectable at $3 \mathrm{~h} \mathrm{PI}$ and is expressed optimally between 6 and $9 \mathrm{~h}$ PI (Legendre et al., 2011). In addition, proteomic study of the purified APMV virion particles showed that both Rab GTPase and PI3K homologs are not associated with the viral particles suggesting that their participation in the early events in establishing infection such as intracellular transport following phagocytosis is unlikely (Renesto et al., 2006). Earlier it was suggested that APMV Rab could be involved in the regulation of host cell cycle (Moreira and Brochier-Armanet, 2008). Here, we speculate that Rab GTPase could also play a role in the viral membrane acquisition during capsid assembly.

\section{A Hypothetical Model for Membrane Acquisition during Mimivirus Capsid Assembly}

The APMV Rab GTPase R214 gene expression starts at $6 \mathrm{~h}$ PI with highest expression at 9h PI (Legendre et al., 2011). Microscopic studies indicated the appearance of viral factory at around $4 \mathrm{~h}$ PI (Suzan-Monti et al., 2007; Mutsafi et al., 2010). The presence of vesicular structures and membrane sheets on the periphery of the viral factory with extensive membrane network are seen at 7.5 h PI (Mutsafi et al., 2013). The small vesicles budding from the ER fuse together to form a multivesicular body (Mutsafi et al., 2013). On the basis of our interaction network analysis, we speculate that the Trans-Golgi network (TGN) could also contribute to the vesicles. The APMV Rab, which is optimally expressed at around the same time, could insert itself into the membrane through its C-terminal prenylation site, get localized near ER and/or TGN and initiate

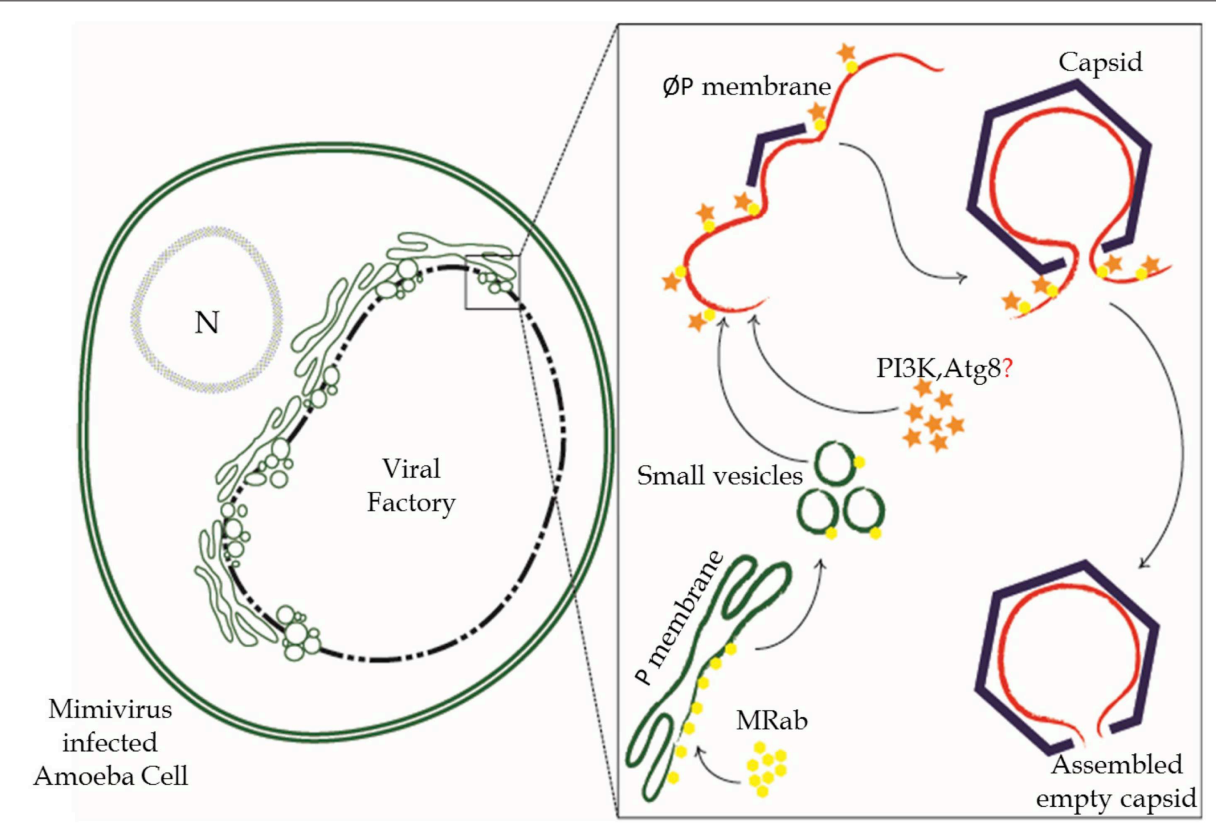

FIGURE 5 | Hypothetical model for the inner membrane acquisition during APMV capsid assembly. The molecular events leading to the inner membrane acquisition for APMV capsid assembly begins with the expression of the Rab GTPase (yellow) at around $6 \mathrm{~h}$ PI. The ER and TGN membrane (green) cisternae gathered at the viral production lines of VF are then subjected to the Rab GTPase mediated pinching of small vesicles. These vesicles fuse together to form a membrane sheet with the help of Rab effectors like PI3K and Atg-8 (orange). The membrane, made amenable through de-phosphorylation (red), serves as a platform for the scaffolding protein and capsid assembly (violet). 
budding of small vesicles (Figure 5). APMV Rab could also facilitate the fusion of smaller vesicles by forming complex with Vps34 and Beclin1. Both Rab5 and Vps34 regulate Atg5-Atg12 conjugation and promote fusion of Atg5-rich phagosome leading to the formation of autophagosomes (Ravikumar et al., 2008). Further, Rab5 mediated recruitment of Atg- 8 that is tethered to the membrane, leads to the hemifusion of small vesicles and expansion of autophagosomes (Nakatogawa et al., 2007). We speculate that the multivesicular bodies observed (Mutsafi et al., 2013) are the result of Atg- 8 mediated fusion of smaller vesicles. These multivesicular bodies fuse and, then rupture leading to the formation of open sheets that are incorporated into the virion. The factors governing the rupture event and further stabilization of the ruptured membrane are yet to be identified (Mutsafi et al., 2013). The Rab GTPase, still bound to the membrane sheet, possibly recruits inositol-5-phosphatase that de-phosphorylates $\mathrm{PI}(4,5) \mathrm{P}_{2}$ of the membrane (Sarantis et al., 2012). The de-phosphorylated membrane is amiable to be molded into any structural form and the scaffolding proteins initiate the expansion of the capsid angular structure over the "softened" membrane (Figure 5) (Chang-Ileto et al., 2011).

The presence of a membrane overhang, lining the assembled nascent capsid, was suggested to prevent the premature sealing of the capsid (Mutsafi et al., 2013). The membrane hang is trimmed after the completion of capsid assembly, leaving a $\sim 20 \mathrm{~nm}$ nonvertex opening (portal) for genome packaging (Mutsafi et al., 2013). The host dynamin protein could mediate the membrane

\section{References}

Ali, B. R., and Seabra, M. C. (2005). Targeting of Rab GTPases to cellular membranes. Biochem. Soc. Trans. 33, 652-656. doi: 10.1042/BST0330652

Bourne, H. R., Sanders, D. A., and McCormick, F. (1990). The GTPase superfamily: a conserved switch for diverse cell functions. Nature 348, 125-132. doi: $10.1038 / 348125 \mathrm{a} 0$

Brumell, J. H., and Scidmore, M. A., (2007). Manipulation of rab GTPase function by intracellular bacterial pathogens. Microbiol. Mol. Biol. Rev. 71, 636-652. doi: 10.1128/MMBR.00023-07

Bucci, C., Lütcke, A., Steele-Mortimer, O., Olkkonen, V. M., Dupree, P., Chiariello, M., et al. (1995). Co-operative regulation of endocytosis by three RAB5 isoforms. FEBS Lett. 366, 65-71. doi: 10.1016/0014-5793(95)00477-Q

Chang-Ileto, B., Frere, S. G., Chan, R. B., Voronov, S. V., Roux, A., and Di Paolo, G. (2011). Synaptojanin 1-mediated PI(4,5)P2 hydrolysis is modulated by membrane curvature and facilitates membrane fission. Dev. Cell 20, 206-218. doi: 10.1016/j.devcel.2010.12.008

Christoforidis, S., Miaczynska, M., Ashman, K., Wilm, M., Zhao, L., Yip, S. C., et al. (1999). Phosphatidylinositol-3-OH kinases are Rab5 effectors. Nat. Cell Biol. 1, 249-252. doi: 10.1038/12075

Claverie, J.-M., Ogata, H., Audic, S., Abergel, C., Suhre, K., and Fournier, P.-E. (2006). Mimivirus and the emerging concept of "giant" virus. Virus Res. 117, 133-144. doi: 10.1016/j.virusres.2006.01.008

Cole, C., Barber, J. D., and Barton, G. J. (2008). The Jpred 3 secondary structure prediction server. Nucleic Acids Res. 36, W197-W201. doi: 10.1093/nar/gkn238

Colson, P., de Lamballerie, X., Fournous, G., and Raoult, D. (2012). Reclassification of giant viruses composing a fourth domain of life in the new order Megavirales. Intervirology 55, 321-332. doi: 10.1159/000336562

DeLano, W. L. (2002). The PyMOL Molecular Graphics System. Schrödinger LLC wwwpymolorg Version 1. Available online at: http://www.pymol.org

Dumas, J. J., Zhu, Z., Connolly, J. L., and Lambright, D. G. (1999). Structural basis of activation and GTP hydrolysis in Rab proteins. Structure 7, 413-423. doi: 10.1016/S0969-2126(99)80054-9 trimming by forming helical oligomers around the inositol lipid giving a tubular morphology to bring about scission (Figure 5, McMahon and Gallop, 2005).

\section{Conclusions}

Our thorough sequence, structural, and phylogenetic analysis showed that the Mimiviridae coded Rab GTPases could constitute a novel isoform Rab5D. In addition, generating an interaction network helped in identifying potential viral and host proteins that might play a role APMV membrane biogenesis. Proposed hypothetical model for APMV membrane biogenesis suggests that intricate interactions between the viral Rab GTPase, other viral coded proteins and several host factors are necessary to bring about APMV membrane biogenesis. These insights gained from the in silico analysis will further aid in understanding the roles of viral and host proteins in APMV membrane biogenesis.

\section{Acknowledgments}

Research in KK lab is supported by grants from BRNS (2012/37B/26/BRNS/1062), DST (SR/SO/BB-0031/2012), DBT (BT/PR4808/BRB/10/1029/2012), and IIT-Bombay seed grant (11IRCCG004). AZ acknowledges Junior Research Fellowship from the University Grants Commission (UGC) and Council of Scientific and Industrial Research (CSIR), India.
Gomes, A. Q., Ali, B. R., Ramalho, J. S., Godfrey, R. F., Barral, D. C., Hume, A. N., et al. (2003). Membrane targeting of Rab GTPases is influenced by the prenylation motif. Mol. Biol. Cell 14, 1882-1899. doi: 10.1091/mbc.E02-10-0639

Gouet, P. (2003). ESPript/ENDscript: extracting and rendering sequence and 3D information from atomic structures of proteins. Nucleic Acids Res. 31, 3320-3323. doi: 10.1093/nar/gkg556

Hinshaw, J. E., and Schmid, S. L. (1995). Dynamin self-assembles into rings suggesting a mechanism for coated vesicle budding. Nature 374, 190-192. doi: 10.1038/374190a0

Ichimura, Y., Kirisako, T., Takao, T., Satomi, Y., Shimonishi, Y., Ishihara, N., et al. (2000). A ubiquitin-like system mediates protein lipidation. Nature 408, 488-492. doi: 10.1038/35044114

Iyer, L. M., Aravind, L., and Koonin, E., V (2001). Common origin of four diverse families of large eukaryotic DNA viruses. J. Virol. 75, 11720-11734. doi: 10.1128/JVI.75.23.11720-11734.2001

Iyer, L. M., Balaji, S., Koonin, E. V., and Aravind, L. (2006). Evolutionary genomics of nucleo-cytoplasmic large DNA viruses. Virus Res. 117, 156-184. doi: 10.1016/j.virusres.2006.01.009

Jones, S. M., Howell, K. E., Henley, J. R., Cao, H., and McNiven, M. A. (1998). Role of dynamin in the formation of transport vesicles from the trans-Golgi network. Science 279, 573-577. doi: 10.1126/science.279.5350.573

Klöpper, T. H., Kienle, N., Fasshauer, D., and Munro, S. (2012). Untangling the evolution of Rab G proteins: implications of a comprehensive genomic analysis. BMC Biol. 10:71. doi: 10.1186/1741-7007-10-71

Kreitzer, G., Marmorstein, A., Okamoto, P., Vallee, R., and Rodriguez-Boulan, E. (2000). Kinesin and dynamin are required for post-Golgi transport of a plasma-membrane protein. Nat. Cell Biol. 2, 125-127. doi: 10.1038/35000081

Kumar, Y., and Valdivia, R. H. (2009). Leading a sheltered life: intracellular pathogens and maintenance of vacuolar compartments. Cell Host Microbe 5, 593-601. doi: 10.1016/j.chom.2009.05.014

La Scola, B., Audic, S., Robert, C., Jungang, L., de Lamballerie, X., Drancourt, M., et al. (2003). A giant virus in amoebae. Science 299, 2033. doi: $10.1126 /$ science. 1081867 
Lee, M.-T. G., Mishra, A., and Lambright, D. G. (2009). Structural mechanisms for regulation of membrane traffic by rab GTPases. Traffic 10, 1377-1389. doi: 10.1111/j.1600-0854.2009.00942.x

Legendre, M., Santini, S., Rico, A., Abergel, C., and Claverie, J.-M. (2011). Breaking the 1000-gene barrier for Mimivirus using ultra-deep genome and transcriptome sequencing. Virol. J. 8:99. doi: 10.1186/1743422X-8-99

Marks, B., Stowell, M. H., Vallis, Y., Mills, I. G., Gibson, A., Hopkins, C. R., et al. (2001). GTPase activity of dynamin and resulting conformation change are essential for endocytosis. Nature 410, 231-235. doi: 10.1038/ 35065645

McGuffin, L. J., Bryson, K., and Jones, D. T. (2000). The PSIPRED protein structure prediction server. Bioinformatics 16, 404-405. doi: 10.1093/bioinformatics/16.4.404

McMahon, H. T., and Gallop, J. L. (2005). Membrane curvature and mechanisms of dynamic cell membrane remodelling. Nature 438, 590-596. doi: 10.1038 /nature04396

Mizushima, N., Noda, T., Yoshimori, T., Tanaka, Y., Ishii, T., George, M. D., et al. (1998). A protein conjugation system essential for autophagy. Nature 395, 395-398. doi: 10.1038/26506

Moore, I., Schell, J., and Palme, K. (1995). Subclass-specific sequence motifs identified in Rab GTPases. Trends Biochem. Sci. 20, 10-12. doi: 10.1016/S09680004(00)88939-2

Moreira, D., and Brochier-Armanet, C. (2008). Giant viruses, giant chimeras: the multiple evolutionary histories of Mimivirus genes. BMC Evol. Biol. 8:12. doi: 10.1186/1471-2148-8-12

Murray, J. T., Panaretou, C., Stenmark, H., Miaczynska, M., and Backer, J. M. (2002). Role of Rab5 in the recruitment of hVps34/p150 to the early endosome. Traffic 3, 416-427. doi: 10.1034/j.1600-0854.2002.30605.x

Mutsafi, Y., Shimoni, E., Shimon, A., and Minsky, A. (2013). Membrane assembly during the infection cycle of the giant Mimivirus. PLoS Pathog. 9:e1003367. doi: 10.1371/journal.ppat.1003367

Mutsafi, Y., Zauberman, N., Sabanay, I., and Minsky, A. (2010). Vaccinia-like cytoplasmic replication of the giant Mimivirus. Proc. Natl. Acad. Sci. U.S.A. 107, 5978-5982. doi: 10.1073/pnas.0912737107

Nakatogawa, H., Ichimura, Y., and Ohsumi, Y. (2007). Atg8, a ubiquitin-like protein required for autophagosome formation, mediates membrane tethering and hemifusion. Cell 130, 165-178. doi: 10.1016/j.cell.2007.05.021

Nei, M., and Kumar, S. (2000). Molecular Evolution and Phylogenetics. New York, NY: Oxford University Press.

Pereira-Leal, J. B., and Seabra, M. C. (2000). The mammalian Rab family of small GTPases: definition of family and subfamily sequence motifs suggests a mechanism for functional specificity in the Ras superfamily. J. Mol. Biol. 301, 1077-1087. doi: 10.1006/jmbi.2000.4010

Pereira-Leal, J. B., and Seabra, M. C. (2001). Evolution of the Rab family of small GTP-binding proteins. J. Mol. Biol. 313, 889-901. doi: 10.1006/jmbi.2001.5072

Pfeffer, S. R. (2005). Structural clues to Rab GTPase functional diversity. J. Biol. Chem. 280, 15485-15488. doi: 10.1074/jbc.R500003200

Rached, F. B., Ndjembo-Ezougou, C., Chandran, S., Talabani, H., Yera, H., Dandavate, V., et al. (2012). Construction of a Plasmodium falciparum Rabinteractome identifies $\mathrm{CK} 1$ and PKA as Rab-effector kinases in malaria parasites. Biol. Cell 104, 34-47. doi: 10.1111/boc.201100081

Raoult, D., Audic, S., Robert, C., Abergel, C., Renesto, P., Ogata, H., et al. (2004). The 1.2-megabase genome sequence of Mimivirus. Science 306, 1344-1350. doi: $10.1126 /$ science. 1101485
Ravikumar, B., Imarisio, S., Sarkar, S., O’Kane, C. J., and Rubinsztein, D. C. (2008). Rab5 modulates aggregation and toxicity of mutant huntingtin through macroautophagy in cell and fly models of Huntington disease. J. Cell Sci. 121, 1649-1660. doi: 10.1242/jcs.025726

Renesto, P., Abergel, C., Decloquement, P., Moinier, D., Azza, S., Ogata, H., et al. (2006). Mimivirus giant particles incorporate a large fraction of anonymous and unique gene products. J. Virol. 80, 11678-11685. doi: 10.1128/JVI.00940-06

Saito, R., Smoot, M. E., Ono, K., Ruscheinski, J., Wang, P.-L., Lotia, S., et al. (2012). A travel guide to Cytoscape plugins. Nat. Methods 9, 1069-1076. doi: 10.1038/nmeth. 2212

Sarantis, H., Balkin, D. M., De Camilli, P., Isberg, R. R., Brumell, J. H., and Grinstein, S. (2012). Yersinia entry into host cells requires Rab5-dependent dephosphorylation of PI(4,5)P2 and membrane scission. Cell Host Microbe 11, 117-128. doi: 10.1016/j.chom.2012.01.010

Sievers, F., Wilm, A., Dineen, D., Gibson, T. J., Karplus, K., Li, W., et al. (2011). Fast, scalable generation of high-quality protein multiple sequence alignments using Clustal Omega. Mol. Syst. Biol. 7, 539. doi: 10.1038/msb.2011.75

Stenmark, H., and Olkkonen, V. M. (2001). The Rab GTPase family. Genome Biol. 2:REVIEWS3007. doi: 10.1186/gb-2001-2-5-reviews3007

Stroupe, C., and Brunger, A. T. (2000). Crystal structures of a Rab protein in its inactive and active conformations. J. Mol. Biol. 304, 585-598. doi: 10.1006/jmbi.2000.4236

Suzan-Monti, M., La Scola, B., Barrassi, L., Espinosa, L., and Raoult, D. (2007). Ultrastructural characterization of the giant volcano-like virus factory of Acanthamoeba polyphaga mimivirus. PLoS ONE 2:e328. doi: 10.1371/journal.pone.0000328

Tamura, K., Stecher, G., Peterson, D., Filipski, A., and Kumar, S. (2013). MEGA6: Molecular evolutionary genetics analysis version 6.0. Mol. Biol. Evol. 30, 2725-2729. doi: 10.1093/molbev/mst197

Woodman, P. G. (2000). Biogenesis of the sorting endosome: the role of Rab5. Traffic 1, 695-701. doi: 10.1034/j.1600-0854.2000.010902.x

Xiao, C., Chipman, P. R., Battisti, A. J., Bowman, V. D., Renesto, P., Raoult, D., et al. (2005). Cryo-electron microscopy of the giant mimivirus. J. Mol. Biol. 353, 493-496. doi: 10.1016/j.jmb.2005.08.060

Yutin, N., and Koonin, E. V. (2009). Evolution of DNA ligases of nucleocytoplasmic large DNA viruses of eukaryotes: a case of hidden complexity. Biol. Direct 4:51. doi: 10.1186/1745-6150-4-51

Zauberman, N., Mutsafi, Y., Halevy, D. B., Shimoni, E., Klein, E., Xiao, C., et al. (2008). Distinct DNA exit and packaging portals in the virus Acanthamoeba polyphaga mimivirus. PLoS Biol. 6:e114. doi: 10.1371/journal.pbio.0060114

Zerial, M., and McBride, H. (2001). Rab proteins as membrane organizers. Nat. Rev. Mol. Cell Biol. 2, 107-117. doi: 10.1038/35052055

Zhang, Y. (2008). I-TASSER server for protein 3D structure prediction. BMC Bioinformatics 9:40. doi: 10.1186/1471-2105-9-40

Conflict of Interest Statement: The authors declare that the research was conducted in the absence of any commercial or financial relationships that could be construed as a potential conflict of interest.

Copyright (c) 2015 Zade, Sengupta and Kondabagil. This is an open-access article distributed under the terms of the Creative Commons Attribution License (CC BY). The use, distribution or reproduction in other forums is permitted, provided the original author(s) or licensor are credited and that the original publication in this journal is cited, in accordance with accepted academic practice. No use, distribution or reproduction is permitted which does not comply with these terms. 


\section{Appendix}

\section{Accession Numbers}

Figure 1:

gi|11931713|162849299, gi|659666264, gi|392056049, gi|31197 7705, gi|557951747, gi|371943930, gi|363540521, gi|448825558, gi|371944856, gi|441432435, gi|310831487, gi|190359301, gi|585299378, gi|211956319, gi|325557805, gi| 160857947 .

\section{Figure 3A:}

gi|82050818: Acanthamoeba polyphaga mimivirus, gi|441432178: Acanthamoeba polyphaga moumouvirus, gi|371943588: Megavirus courdo7, gi|123471026: Trichomonas vaginalis G3, gi|471227609: Ichthyophthirius multifiliis, gi|403370178: Oxytricha trifallax, gi|159024328: Aiptasia pulchella, gi|62736284: Trichomonas vaginalis, gi|225711860: Lepeophtheirus salmonis, gi|358338265: Clonorchis sinensis, gi|511004806: Mucor circinelloides $\mathrm{f}$. circinelloides $1006 \mathrm{PhL}$, gi|31745716: Toxoplasma gondii, gi|675134504: Hammondia hammondi, gi|641579338: Plasmodium reichenowi, gi|124512956: Plasmodium falciparum 3D7, gi|470308728: Capsaspora owczarzaki ATCC 30864, gi|457876654: Plasmodium cynomolgi strain B, gi|672186478: Plasmodium inui San Antonio 1, gi|15986733: Mus musculus, gi|168001170: Physcomitrella patens, gi|221060859: Plasmodium knowlesi strain H, gi|307777770: Tetrahymena thermophila, gi|118355342: Tetrahymena thermophila, gi|168056370: Physcomitrella patens, gi|353232500: Schistosoma mansoni, gi|54792729: Canis lupus familiaris, gi|33150586: Homo sapiens, gi|675122722: Hammondia hammondi, gi|8394136: Rattus norvegicus, gi|148692254: Mus musculus, gi|431920194: Pteropus alecto, gi|237831345: Toxoplasma gondii ME49, gi|401407536: Neospora caninum Liverpool, gi|221061061: Plasmodium knowlesi strain H, gi|156102877: Plasmodium vivax Sal-1, gi|318270757: Ictalurus punctatus, gi|457876942: Plasmodium cynomolgi strain B, gi|74834378: Paramecium tetraurelia, gi|325190342: Albugo laibachii Nc14, gi|242021638: Pediculus humanus corporis, gi|74834500: Paramecium tetraurelia, gi|168062057: Physcomitrella patens, gi|241643394: Ixodes scapularis, gi|557240148: Eimeria maxima, gi|294895479: Perkinsus marinus ATCC 50983, gi|118372409: Tetrahymena thermophila, gi|57524538: Danio rerio, gi|646712526: Zootermopsis nevadensis, gi|557230549: Eimeria necatrix, gi|729714020: Rhizopus microsporus, gi|577151129: Plasmodium vinckei petteri, gi|124513178: Plasmodium falciparum 3D7, gi|225717876: Caligus clemensi, gi|225710156: Caligus rogercresseyi, gi|672186872: Plasmodium inui San Antonio 1, gi|562974055: Ogataea parapolymorpha DL1, gi|675234676: Plasmodium yoelii, gi|661187471: Lichtheimia corymbifera JMRC:FSU:9682, gi|661186371: Lichtheimia corymbifera JMRC:FSU:9682, gi|74025730: Trypanosoma brucei brucei TREU927, gi|68075387: Plasmodium berghei strain ANKA, gi|749164803: Gregarina niphandrodes, gi|675228811:Plasmodium berghei ANKA, gi|703115059: Morus notabilis, gi|727139470: Rhizopus microspores, gi|444732045: Tupaia chinensis, gi|669196256: Plasmodium vinckei vinckei, gi|473785860: Triticum urartu, gi|675223050: Plasmodium chabaudi chabaudi, gi|294887922: Perkinsus marinus ATCC 50983, gi|167384556: Entamoeba dispar SAW760, gi|148922827:
Danio rerio, gi|730370459: Trichuris suis gi|470391604: Acanthamoeba castellanii str. Neff, gi|357625222: Danaus plexippus, gi|124088730: Paramecium tetraurelia strain d4-2, gi|325191836: Albugo laibachii Nc14, gi|118387620: Tetrahymena thermophila, gi|224069898: Populus trichocarpa, gi|218204: Oryza sativa Japonica Group, gi|62897579: Homo sapiens, gi|669196060: Plasmodium vinckei vinckei, gi|89258415: Suberites domuncula, gi|528272994: Angomonas deanei, gi|557239031: Eimeria necatrix, gi|77404180: Rattus norvegicus, gi|62859319: Xenopus (Silurana) tropicalis, gi|67475925: Entamoeba histolytica HM1:IMSS, gi|168040993: Physcomitrella patens.

\section{Figure 3B:}

Homo sapiens (HS)

sp|P62491， sp|P20340, sp|P51159, sp|Q13636， sp|Q15286, sp|Q86YS6, sp|Q9BZG1, sp|Q9NP90, sp|P61006, sp|P20338, sp|P20339, sp|P51151, sp|P51149, sp|P62820, sp|P61019, sp|Q9UL26, sp|Q14964, sp|Q96QF0, sp|Q9H0N0, sp|P51148, sp|O95716, sp|O14966, sp|Q15907, sp|Q9H0U4, sp|Q9ULC3, sp|P61106, sp|P51153, sp|Q9NRW1， sp|Q9UL25, sp|P61026, sp|Q9NP72, sp|Q6IQ22， sp|Q13637， sp|P61020， sp|P51157, sp|Q99P58， sp|A4D1S5， sp|Q969Q5， sp|Q96AH8， sp|P57729, sp|Q15771， sp|O95755， sp|P57735， sp|O00194， sp|P61018, sp|P20337, sp|Q8WUD1, sp|P20336, sp|Q92930, sp|Q9ULW5, sp|Q9H082，sp|Q9H0T7，sp|Q96DA2，sp|Q96AX2，sp|P59190, sp|Q96E17, sp|Q8WXH6, sp|Q96S21， sp|Q14088， sp|Q5JT25, sp|Q9NX57, sp|Q12829, sp|Q7Z6P3.

\section{Plasmodium falciparum (PF)}

$\operatorname{tr}|\mathrm{Q} 8 \mathrm{I} 274, \operatorname{tr}| \mathrm{Q} 7 \mathrm{~K} 6 \mathrm{~A} 8, \operatorname{tr}|\mathrm{Q} 8 \mathrm{IHR} 8, \operatorname{tr}| \mathrm{Q} 76 \mathrm{NM} 4, \operatorname{tr} \mid \mathrm{Q} 7 \mathrm{~K} 6 \mathrm{~B} 0$, $\operatorname{tr}|\mathrm{O} 96193, \operatorname{tr}| \mathrm{Q} 8 \mathrm{I} 5 \mathrm{~A} 9, \operatorname{tr}|\mathrm{C} 0 \mathrm{H} 5 \mathrm{G} 2, \operatorname{tr}| \mathrm{Q} 76 \mathrm{NM} 7, \operatorname{tr} \mid \mathrm{Q} 8 \mathrm{I} 3 \mathrm{~W} 9$, $\operatorname{tr} \mid \mathrm{C} 0 \mathrm{H} 516$.

Caenorhabditis elegans (CE)

sp|Q94986, sp|Q8MXS1， sp|P34213， sp|Q20365， sp|Q22782,

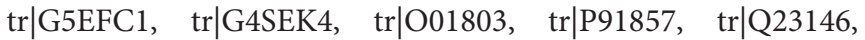
$\operatorname{tr}|\mathrm{Q} 95 \mathrm{QV} 3, \operatorname{tr}| \mathrm{Q} 9 \mathrm{U} 2 \mathrm{C3}, \operatorname{tr}|\mathrm{Q} 1 \mathrm{ZXR} 4, \operatorname{tr}| \mathrm{Q} 93874, \operatorname{tr} \mid \mathrm{Q} 9 \mathrm{XWZ3}$, $\operatorname{tr}|\mathrm{Q} 22045, \operatorname{tr}| \mathrm{Q} 18969, \operatorname{tr}|\mathrm{Q} 9 \mathrm{U} 1 \mathrm{~W} 9, \operatorname{tr}| \mathrm{Q} 94148, \operatorname{tr} \mid \mathrm{Q} 9 \mathrm{UAQ} 6$, $\operatorname{tr} \mid$ G5EFA6, tr|Q9XWR6, tr|Q6BCV5.

Drosophila melanogaster (DM)

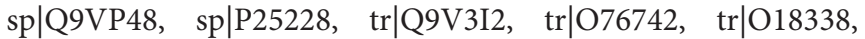
$\operatorname{tr}|\mathrm{O} 18339, \operatorname{tr}| \mathrm{Q} 7 \mathrm{KY} 04, \operatorname{tr}|\mathrm{Q} 9 \mathrm{VM} 50, \operatorname{tr}| \mathrm{O} 18336, \operatorname{tr} \mid \mathrm{O} 18332$, $\operatorname{tr} \mid$ Q9W3Q0, $\operatorname{tr} \mid$ Q9W585, $\operatorname{tr} \mid$ Q9W5X0, tr|Q8IPT6, $\operatorname{tr} \mid \mathrm{O} 18335$, $\operatorname{tr} \mid$ O76901, $\operatorname{tr}|\mathrm{Q} 9 \mathrm{~W} 4 \mathrm{~A} 0, \operatorname{tr}| \mathrm{Q} 9 \mathrm{~W} 2 \mathrm{~S} 9, \operatorname{tr}|\mathrm{O} 18333, \operatorname{tr}| \mathrm{Q} 7 \mathrm{PLE} 9$, $\operatorname{tr}|\mathrm{O} 18334, \operatorname{tr}| \mathrm{O} 15971, \operatorname{tr}|\mathrm{Q} 86 \mathrm{BK} 7, \operatorname{tr}| \mathrm{Q} 86 \mathrm{BK} 8, \operatorname{tr} \mid \mathrm{Q} 9 \mathrm{VIW6}$, $\operatorname{tr} \mid$ Q8IR98, $\operatorname{tr} \mid$ Q9VNG6, $\operatorname{tr|Q8IR80,~} \operatorname{tr|F0JAF7,~} \operatorname{tr} \mid$ Q7PLE8, $\operatorname{tr} \mid$ Q8IRK9, $\operatorname{tr} \mid$ Q8IRK8, $\operatorname{tr} \mid$ Q9VYM8, $\operatorname{tr} \mid$ Q9VZ47, $\operatorname{tr} \mid$ Q95RQ2, $\operatorname{tr} \mid \mathrm{Q} 8$ SYS5, $\operatorname{tr}|\mathrm{Q} 9 \mathrm{~V} 3 \mathrm{C} 2, \operatorname{tr}| \mathrm{M} 9 \mathrm{NEE} 8, \operatorname{tr}|\mathrm{M} 9 \mathrm{PG} 39, \operatorname{tr}| \mathrm{O} 18337$, $\operatorname{tr}|\mathrm{Q} 8 \mathrm{~T} 046, \operatorname{tr}| \mathrm{Q} 95 \mathrm{RH} 7, \operatorname{tr}|\mathrm{B} 8 \mathrm{~A} 3 \mathrm{X} 3, \operatorname{tr}| \mathrm{Q} 8 \mathrm{IGH} 2, \operatorname{tr} \mid \mathrm{Q} 8 \mathrm{MSX}$, $\operatorname{tr|Q8MRA4,~tr|Q9VC31,~} \operatorname{tr|Q9W2V3.~}$

Trichomonas vaginalis (TV)

$\operatorname{tr}|\mathrm{Q} 4 \mathrm{FIE} 4, \operatorname{tr}| \mathrm{Q} 4 \mathrm{G} 290, \operatorname{tr} \mid \mathrm{Q} 3$ YE89, $\operatorname{tr} \mid \mathrm{Q} 3$ YE90, $\operatorname{tr} \mid \mathrm{Q} 4 \mathrm{G} 2 \mathrm{~B} 4$, $\operatorname{tr}|\mathrm{Q} 4 \mathrm{G} 2 \mathrm{~B} 7, \operatorname{tr}| \mathrm{Q} 4 \mathrm{G} 298$.

Saccharomyces cerevisiae (SC)

sp|P01123， sp|P38555, sp|P51996, sp|P48559, sp|P36018, sp|P38146, sp|P32939, sp|Q99260. 